\title{
Plasma C3 and C3a Levels in Cryptogenic and Large-Vessel Disease Stroke: Associations with Outcome
}

\author{
Anna Stokowska Sandra Olsson Lukas Holmegaard Katarina Jood \\ Christian Blomstrand Christina Jern Marcela Pekna \\ Institute of Neuroscience and Physiology, Department of Clinical Neuroscience and Rehabilitation, \\ The Sahlgrenska Academy at University of Gothenburg, Gothenburg, Sweden
}

\section{Key Words}

Complement system $\cdot \mathrm{C} 3 \cdot \mathrm{C} 3 \mathrm{a} \cdot$ Ischemic stroke $\cdot$ Outcome

\begin{abstract}
Background and Purpose: Inflammation seems to be a key player in the pathophysiology of stroke. In this study, we compared plasma C3 and C3a levels in cryptogenic and large-vessel disease (LVD) subtypes of ischemic stroke and control subjects and evaluated their association to outcome at 3 months and 2 years. Methods: C3 and C 3 a levels in plasma of 79 cryptogenic stroke and 73 LVD stroke patients, sampled within 10 days and at 3 months after stroke, and ageand sex-matched control subjects from the Sahlgrenska Academy Study on Ischemic Stroke were measured by ELISA. Functional outcome was assessed with the modified Rankin Scale. Results: Plasma C3 was increased in both stroke groups at both time points. Systemic elevation of C3a was limited to the acute phase in the cryptogenic stroke group, whereas plasma C3a levels in the LVD group were also elevated at the 3-month follow-up. In the LVD group, plasma C3 levels in the upper third at the 3-month follow-up were associated with an unfavorable outcome after 3 months independently of age and sex: odds ratio (OR) 5.56; 95\% confidence interval (Cl) 1.03-29.93; $p=0.045$; as well as after 2 years: OR $4.75 ; 95 \% \mathrm{Cl} 1.11-20.30 ; \mathrm{p}=0.036$. In the crypto-
\end{abstract}

genic stroke group, high plasma C3a levels in the acute phase were associated with an unfavorable outcome after 3 months: OR 3.75; 95\% Cl 1.01-13.96; $p=0.049$ in univariate analysis but not after adjustment for age and sex $(p=0.050)$. Conclusions: Plasma C3 and C3a levels are elevated in cryptogenic and LVD stroke and the predictive value of these markers may depend on stroke subtype. Further studies on the role of the complement system in ischemic stroke outcome based on larger patient populations and controlling for the effect of infections, are clearly warranted.

Copyright $\odot 2011$ S. Karger AG, Basel

\section{Introduction}

Inflammation seems to play a key role in the pathophysiology of stroke $[1,2]$ as well as in the development and destabilization of atherosclerotic plaques $[3,4]$, and progression of symptomatic intracranial large-vessel disease (LVD) is associated with a proinflammatory state [5]. Plasma levels of several inflammatory markers have recently been shown to be altered during the first 7 days after ischemic stroke and to be independently related to clinical outcome scores on day 90 [6]. The complement system is a major inflammatory mediator that appears to play a dual role in experimental stroke. Complement

\section{KARGER}

Fax +41613061234 E-Mail karger@karger.ch www.karger.com
(C) 2011 S. Karger AG, Base

$1015-9770 / 11 / 0322-0114 \$ 38.00 / 0$

Accessible online at:

www.karger.com/ced
Dr. Marcela Pekna, MD, PhD

Institute of Neuroscience and Physiology, Department of Clinical Neuroscience and

Rehabilitation, The Sahlgrenska Academy at University of Gothenburg

Medicinaregatan 9a, Box 440, SE-405 30 Göteborg (Sweden)

Tel. +46 31786 3581, E-Mail Marcela.Pekna@ @euro.gu.se 
plays a positive role in normal CNS maintenance and genetic deficiency of the third complement component (C3) was associated with larger cerebral infarcts after permanent focal brain ischemia [7]. Anaphylatoxic peptides C3a and $\mathrm{C} 5 \mathrm{a}$, generated in the process of complement activation, can be protective against excitotoxicity-induced neuronal death and can ultimately contribute to healing $[8,9]$. On the other hand, inhibition of complement activation reduced infarct volume and neurological impairments after transient cerebral ischemia in animal models [10-13]. In humans, the role of complement in ischemic stroke is less clear. Complement is activated locally in the human brain in the course of ischemic stroke as the deposits of both early complement components (C1q, C3c and C4d) and terminal complex (C5b-9) were found in the infarcts $[14,15]$. Plasma levels of C5b-9, C4d, C3 and $\mathrm{C} 3 \mathrm{a}$ were shown to be elevated in the acute phase of ischemic stroke [16-18] and the levels of soluble C5b-9 positively correlated with the clinical severity of stroke, the degree of neurological deficit and functional disability 6 days after admission [16]. However, these studies involved only small patient groups that did not allow for separate analysis of complement system response in individual stroke subtypes.

In the present study, we compared plasma levels of C3 and its activation product $\mathrm{C} 3 \mathrm{a}$ in control subjects and in two etiologically different ischemic stroke subtypes, namely LVD and cryptogenic stroke. We investigated the association of plasma C3 and C3a levels with stroke, correlations with high-sensitivity C-reactive protein (hsCRP), and functional outcome at 3 months and 2 years after admission.

\section{Subjects and Methods}

\section{Study Population}

The study population comprises participants in the Sahlgrenska Academy Study on Ischemic Stroke (SAHLSIS) and population-based controls free from stroke and clinical atherothrombotic disease [19]. In short, patients who presented with first-ever or recurrent acute ischemic stroke before reaching the age of 70 years were consecutively recruited $(n=600)$. Healthy community Caucasian controls $(n=600)$ were randomly selected as described. All patients underwent neuroimaging and were examined by a physician, both on admission and after 3 months. The patients were classified into stroke subtypes according to the Trial of Org 10172 in Acute Stroke Treatment (TOAST) criteria. The distribution of stroke etiologic subtypes in the population was as follows: LVD ( $\mathrm{n}=73,12 \%)$, small-vessel disease $(\mathrm{n}=124,21 \%)$; cardioembolic stroke ( $n=98,16 \%)$; cryptogenic stroke $(n=162,27 \%)$; other determined stroke $(\mathrm{n}=51,9 \%)$, and undetermined stroke $(\mathrm{n}=$ 92, 15\%). Cryptogenic stroke was diagnosed when no cause was identified despite extensive evaluation. Undetermined stroke included cases in which more than one cause was identified or when the evaluation was cursory. Maximum stroke severity within the first 10 days after the stroke was scored using the Scandinavian Stroke Scale (SSS). SSS is a validated measure of neurological impairment. However, it should be noted that it differs in the direction of measurement compared to the National Institutes of Health Stroke Scale (NIHSS), e.g. no impairment is 58 of 58 on the SSS and 0 of 42 on the NIHSS. Functional outcome after 3 months and 2 years was assessed with the modified Rankin Scale (mRS).

In the present study, we investigated ischemic stroke due to atherosclerosis, i.e. LVD, and stroke due to an unknown cause, i.e. cryptogenic stroke. To this end, all patients classified as having stroke due to $\operatorname{LVD}(\mathrm{n}=73)$ and a similar number of patients with cryptogenic stroke $(n=79$, i.e. half of the patients classified as having this subtype) were included. Because the cryptogenic group has a relatively favorable functional outcome, all cryptogenic stroke patients with an $\mathrm{mRS}$ score $>2$ were selected $(\mathrm{n}=31)$ to minimize the effect of uneven distribution of outcome categories in this subgroup and thus improve the statistical power in the outcome regression analysis. The other 48 cryptogenic stroke patients were selected so that they represented an even distribution of the mRS scores 0,1 and 2, i.e. a similar number of patients with each score was randomly selected. For each of the two groups, half the number of controls (selected so as to have the same mean age and sex distribution as the cases) was analyzed.

The study was approved by the Ethical Committee of the University of Gothenburg. All participants provided written informed consent prior to enrolment. For those participants who were unable to communicate, consent was obtained from the next of kin.

\section{Blood Sampling}

In patients, blood sampling was performed within 10 days of the stroke event (median day of sampling was 4.5 and 5.0 after the stroke event, for the cryptogenic and LVD group, respectively) and at the 3-month follow-up. In controls, blood sampling was performed once. Venous blood was collected in ethylenediaminetetraacetic acid (EDTA-Vacuette tubes, Greiner) between 8:30 and 10:30 a.m. after an overnight fast, centrifuged and supernatants were stored at $-80^{\circ} \mathrm{C}$. Serum levels of hsCRP were analyzed as reported previously [20].

\section{C3 and C3a Measurements}

Plasma C3 was measured by sandwich ELISA. Wells of microtiter plates were coated with polyclonal rabbit anti-C3c antibody (Dako, Glostrup, Denmark) in phosphate-buffered saline at $4^{\circ} \mathrm{C}$ overnight. The plates were saturated with dilution buffer, i.e. phosphate-buffered saline containing 0.05\% (v/v) Tween 20 (Sigma, St. Louis, Mo., USA) and 1\% bovine serum albumin (Sigma) for $30 \mathrm{~min}$ at room temperature. Sample or human C3 (Sigma), used as a standard, was incubated for $60 \mathrm{~min}$ at room temperature. Horseradish peroxidase-conjugated polyclonal anti-C3c antibody (Dako) was incubated for $60 \mathrm{~min}$ at room temperature. After each incubation step, the plates were washed with PBS containing $0.05 \%(\mathrm{v} / \mathrm{v})$ Tween 20 . The enzyme reaction was started by addition of color reagent: $20 \mathrm{mg}$ of 1,2-phenylendiamine-dihydrochloride (Sigma), and $10 \mu \mathrm{H}_{2} \mathrm{O}_{2}$ in $75 \mathrm{ml} 0.1 \mathrm{M}$ citrate/phosphate buffer, $\mathrm{pH}$ 5. The reaction was stopped by addition of $1 \mathrm{M}$ $\mathrm{H}_{2} \mathrm{SO}_{4}$ after $3 \mathrm{~min}$. The staining was quantified spectrophotometrically at $490 \mathrm{~nm}$. Plasma samples were analyzed for C3a with 
Table 1. Demographic and clinical characteristics of the study population

\begin{tabular}{|c|c|c|c|c|}
\hline & \multicolumn{2}{|l|}{ Cryptogenic stroke } & \multicolumn{2}{|l|}{ LVD stroke } \\
\hline & controls $(n=40)$ & patients $(n=79)$ & controls $(n=39)$ & patients $(n=73)$ \\
\hline Median age, years & $59(53-62)$ & $57(51-63)$ & $60(56-63)$ & $60(57-64)$ \\
\hline Male sex, $\mathrm{n}$ & $25(62)$ & $49(62)$ & $30(77)$ & $54(74)$ \\
\hline Hypertension, $\mathrm{n}$ & $16(40)$ & $49(62)^{*}$ & $14(36)$ & $44(60)^{*}$ \\
\hline Diabetes mellitus, $\mathrm{n}$ & $5(12)$ & $13(16)$ & $5(13)$ & $25(34)^{*}$ \\
\hline Current smoking, $\mathrm{n}$ & $5(12)$ & $31(39)^{* *}$ & $9(23)$ & $39(53)^{* *}$ \\
\hline Hyperlipidemia, $\mathrm{n}$ & $21(52)$ & $53(69)$ & $29(74)$ & $53(82)$ \\
\hline BMI, median & $25.38(22.29-28.50)$ & $27.71(24.03-28.23)$ & $26.52(25.33-28.91)$ & $26.12(24.05-28.91)$ \\
\hline SSS acute, median & - & $52(31-55)$ & - & $52(33-57)$ \\
\hline \multicolumn{5}{|l|}{ hsCRP, median, mg/l } \\
\hline Acute & $1.98(0.89-4.13)$ & $2.76(1.11-7.91)$ & $1.49(0.96-2.37)$ & $4.66(1.86-13.28)^{* * *}$ \\
\hline Follow-up & - & $1.81(1.03-4.24)$ & - & $3.64(1.58-9.28)^{* * *}$ \\
\hline
\end{tabular}

Figures shown in parentheses are percentages (male sex, hypertension, diabetes mellitus, current smoking and hyperlipidemia) or interquartile ranges (median age, BMI, SSS acute and hsCRP).

Differences between cases and controls were examined with the $\chi^{2}$ test for proportions or the Mann-Whitney U test for continuous variables. $\mathrm{LVD}=$ Large-vessel disease stroke; $\mathrm{BMI}=$ body mass index; SSS = Scandinavian Stroke Scale score; hsCRP = high sensitivity C-reactive protein. ${ }^{*} \mathrm{p}<0.05 ;{ }^{* *} \mathrm{p}<0.01 ;{ }^{* * *} \mathrm{p}<0.001$.

an ELISA kit (Quidel Corporation, San Diego, Calif., USA) according to the manufacturer's recommendations.

\section{Statistical Analysis}

Due to a predominantly non-Gaussian distribution of C3 and C3a values, nonparametric tests were employed for the statistical analysis. Differences in characteristics between cases and controls were examined with the $\chi^{2}$ test for proportions for categorical variables and the Mann-Whitney $U$ test for continuous variables. Plasma levels of C3 and C3a are presented as medians and interquartile ranges. Time point differences of C3 and C3a levels were compared using the Wilcoxon Signed Rank test. Correlations between plasma levels of C3, C3a and serum levels of hsCRP were estimated by Spearman's rank correlation coefficient, with a two-tailed significance test. For the regression models of the association with stroke, values for the $\mathrm{C} 3$ and $\mathrm{C} 3$ a levels were standardized using the means and standard deviations of the respective control population. Furthermore, the values of complement protein levels were divided into tertiles.

The association between complement levels and case/control status was investigated with binary logistic regression adjusted for the established risk factors, i.e. age, sex, hypertension, smoking status and diabetes mellitus (model 1). For studying a possible association of complement levels and functional outcome at 3 months and 2 years after stroke (favorable, mRS 0-2 vs. unfavorable, mRS 3-6), a regression model adjusted for age and sex (model 2) was used. Odds ratios (ORs) and 95\% confidence intervals (CIs) were calculated separately for the two ischemic stroke subtypes, where patient groups were compared with their respective control group.

The cryptogenic and LVD group samples were analyzed on two separate occasions more than a year apart. Therefore, to avoid erroneous conclusions resulting from a possible change in re- agents and plasma sample stability between the two occasions, direct comparisons were performed only between patients and their simultaneously analyzed controls but not between the subtype groups.

Statistical analysis was performed using $\mathrm{R}$ statistical package 2.10.1. Adjustment for multiple testing was not conducted as our study was considered to be hypothesis generating.

\section{Missing Values}

The numbers of individuals with missing values were as follows: smoking status, $\mathrm{n}=2$; hypertension, $\mathrm{n}=3$; SSS score, $\mathrm{n}=4$; hyperlipidemia, $\mathrm{n}=10$; hsCRP, $\mathrm{n}=14$ (acute, $\mathrm{n}=5$, follow-up, $\mathrm{n}=$ 9), C3, $n=17$ (acute, $n=6$, follow-up, $n=11$ ), C3a, $n=17$ (acute, $\mathrm{n}=6$, follow-up, $\mathrm{n}=11$ ) and $\mathrm{mRS}, \mathrm{n}=7$ ( 3 months, $\mathrm{n}=5$, 2 years, $\mathrm{n}=2$ ). In the regression analysis, for the categorical variables with missing values, respective dummy variables were created and included in the model. Dummy was coded 1 when the value of a parameter was missing and 0 when the value was present. These variables were not significant at any point $(p>0.97)$, indicating that missing values did not affect the results of regression analysis.

The manuscript was prepared according to the STROBE guidelines [21].

\section{Results}

Demographic and clinical characteristics of the patient and control groups whose samples were used for the analysis of the plasma levels of $\mathrm{C} 3$ and $\mathrm{C} 3 \mathrm{a}$ are summarized in table 1. Hypertension and smoking were 

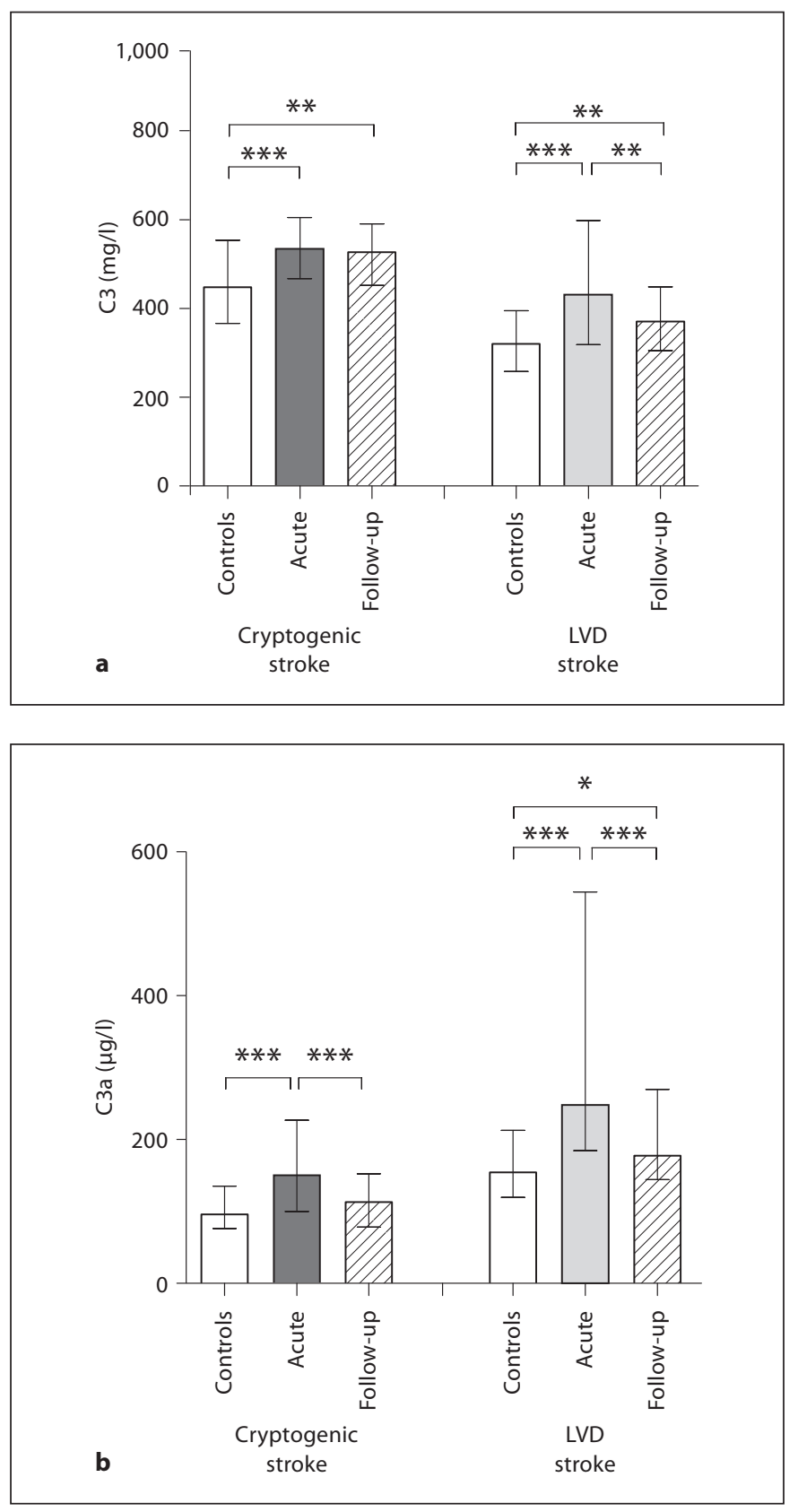

Fig. 1. Median levels of plasma C3 (a) and C3a (b), with error bars representing interquartile ranges. Differences between control and acute or follow-up measurements were examined by the Mann-Whitney $U$ test and acute versus follow-up, by the Wilcoxon signed rank test. ${ }^{*} \mathrm{p}<0.05 ;{ }^{* *} \mathrm{p}<0.01 ;{ }^{* *} \mathrm{p}<0.001$.

more common in cases than in controls; diabetes was more common in patients with LVD stroke than in the corresponding control subjects. The LVD group also displayed significantly higher levels of hsCRP compared to controls.
Table 2. Correlations between plasma levels of $\mathrm{C} 3$ and $\mathrm{C} 3 \mathrm{a}$, hsCRP, and SSS

\begin{tabular}{lllll}
\hline & $\begin{array}{l}\text { C3a } \\
\text { follow-up }\end{array}$ & C3 acute & hsCRP & $\begin{array}{l}\text { SSS } \\
\text { acute }\end{array}$ \\
\hline $\begin{array}{l}\text { Cryptogenic stroke } \\
\text { C3 acute }\end{array}$ & - & - & $0.446^{* * *}$ & -0.203 \\
C3 follow-up & -0.078 & $0.670^{* * *}$ & $0.364^{* *}$ & -0.138 \\
C3a acute & $0.502^{* *}$ & $-0.236^{*}$ & 0.167 & -0.043 \\
C3a follow-up & - & - & $0.278^{*}$ & -0.070 \\
LVD stroke & & & & \\
C3 acute & - & - & 0.136 & -0.024 \\
C3 follow-up & 0.141 & $0.438^{* * *}$ & 0.141 & $-0.307^{*}$ \\
C3a acute & $0.526^{* * *}$ & $0.527^{* * *}$ & $0.376^{* *}$ & -0.070 \\
C3a follow-up & - & - & $0.251^{*}$ & 0.101 \\
Controls & & & & \\
C3 & - & - & $0.316^{* *}$ & - \\
C3a & - & -0.220 & 0.043 & - \\
\hline
\end{tabular}

Values given represent Spearman's correlation coefficients $(\rho)$ with significance levels denoted as follows: ${ }^{*} \mathrm{p}<0.05$; ${ }^{* *} \mathrm{p}<0.01$; ${ }^{* * *} \mathrm{p}<0.001$. $-=$ Nonsignificant correlation; hsCRP $=$ high sensitivity C-reactive protein; SSS = Scandinavian Stroke Scale score; LVD = large-vessel disease .

${ }^{1}$ Variable measured at the same time point as the respective indicated measurements of C3 or C3a.

\section{Plasma C3 and C3a Levels after Ischemic Stroke}

In both the cryptogenic and LVD stroke groups, the plasma C3 levels at both time points were elevated compared to the control groups. The $\mathrm{C} 3$ levels in the acute phase were significantly higher compared to the followup samples in the LVD group ( $<<0.01)$, whereas in the cryptogenic stroke group the C3 levels at the 3-month follow-up did not differ from those in the acute phase (fig. 1a). In the acute phase, the plasma levels of C3a were higher in patients than in the control groups in both stroke subtypes $(\mathrm{p}<0.001)$. At the 3 -month follow-up, the C3a levels declined in both stroke subtype groups $(\mathrm{p}<$ 0.001); however, in the LVD group, the C3a levels were still higher than the control levels $(\mathrm{p}<0.05$; fig. $1 \mathrm{~b})$.

The C3 levels were positively correlated with hsCRP at both time points in the cryptogenic group. In this group, the plasma $\mathrm{C} 3 \mathrm{a}$ levels in the acute phase correlated negatively with the $\mathrm{C} 3$, and the $\mathrm{C} 3 \mathrm{a}$ levels at the 3-month follow-up were positively correlated with hsCRP. In the LVD group, the acute plasma C3a levels were positively correlated with the $\mathrm{C} 3$ and hsCRP levels; the plasma C3a levels at the 3-month follow-up correlated with hsCRP (table 2). 
Table 3. Univariate and multivariate ORs with $95 \%$ CIs of association between C 3 and C 3 a levels and stroke

\begin{tabular}{|c|c|c|c|c|}
\hline Tertiles of C3 and C3a & Controls, $\mathrm{n}$ & Cases, $\mathrm{n}$ & Univariate OR & Multivariate model $1 \mathrm{OR}$ \\
\hline \multicolumn{5}{|l|}{ Cryptogenic stroke } \\
\hline $\mathrm{C} 3$ acute, all & 40 & 75 & & \\
\hline Lower third & 22 & 18 & 1.0 (reference) & 1.0 (reference) \\
\hline Middle third & 9 & 26 & $3.53(1.32-9.42)^{*}$ & $3.95(1.32-11.80)^{*}$ \\
\hline Upper third & 9 & 31 & $4.21(1.60-11.09)^{* *}$ & $4.45(1.51-12.87)^{* *}$ \\
\hline C3 follow-up, all & 40 & 77 & & \\
\hline Lower third & 21 & 19 & 1.0 (reference) & 1.0 (reference) \\
\hline Middle third & 9 & 28 & $3.44(1.30-9.11)^{*}$ & $4.34(1.48-12.75)^{* *}$ \\
\hline Upper third & 10 & 30 & $3.32(1.29-8.55)^{*}$ & $2.70(0.94-7.71)$ \\
\hline C3a acute, all & 40 & 75 & & \\
\hline Lower third & 21 & 19 & 1.0 (reference) & 1.0 (reference) \\
\hline Middle third & 13 & 26 & $2.21(0.89-5.49)$ & $2.47(0.87-7.09)$ \\
\hline Upper third & 6 & 30 & $5.53(1.89-16.48)^{* *}$ & $8.99(2.53-31.90)^{* * *}$ \\
\hline C3a follow-up, all & 40 & 77 & & \\
\hline Lower third & 16 & 24 & 1.0 (reference) & 1.0 (reference) \\
\hline Middle third & 12 & 26 & $1.44(0.57-3.67)$ & $1.39(0.43-3.89)$ \\
\hline Upper third & 12 & 27 & $1.50(0.59-3.80)$ & $1.18(0.42-3.27)$ \\
\hline \multicolumn{5}{|l|}{ LVD stroke } \\
\hline C3 acute, all & 39 & 71 & & \\
\hline Lower third & 19 & 19 & 1.0 (reference) & 1.0 (reference) \\
\hline Middle third & 14 & 23 & $1.64(0.66-4.12)$ & $1.91(0.64-5.71)$ \\
\hline Upper third & 6 & 29 & $4.83(1.63-14.30)^{* *}$ & $5.93(1.67-20.87)^{* *}$ \\
\hline C3 follow-up, all & 39 & 65 & & \\
\hline Lower third & 19 & 19 & 1.0 (reference) & 1.0 (reference) \\
\hline Middle third & 10 & 19 & $1.80(0.66-4.89)$ & $1.96(0.60-6.42)$ \\
\hline Upper third & 10 & 27 & $2.70(1.03-7.09)^{*}$ & $3.26(1.04-10.23)^{*}$ \\
\hline C3a acute, all & 39 & 71 & & \\
\hline Lower third & 23 & 15 & 1.0 (reference) & 1.0 (reference) \\
\hline Middle third & 12 & 25 & $3.19(1.24-8.23)^{*}$ & $2.46(0.84-7.19)$ \\
\hline Upper third & 4 & 31 & $11.88(3.48-40.56)^{* * *}$ & $11.55(2.98-44.79)^{* * *}$ \\
\hline C3a follow-up, all & 39 & 64 & & \\
\hline Lower third & 19 & 19 & 1.0 (reference) & 1.0 (reference) \\
\hline Middle third & 12 & 22 & $1.83(0.71-4.73)$ & $2.05(0.69-7.16)$ \\
\hline Upper third & 8 & 23 & $2.87(1.03-8.02)^{*}$ & $3.72(1.16-11.94)^{*}$ \\
\hline
\end{tabular}

Figures shown in parentheses are 95\% CIs. Model 1: adjusted for age, sex, hypertension, smoking and diabetes; ${ }^{*} \mathrm{p}<0.05 ;{ }^{* *} \mathrm{p}<$ $0.01 ;{ }^{* *} \mathrm{p}<0.001$. OR $=$ Odds ratio; $\mathrm{CI}=$ confidence interval; $\mathrm{LVD}=$ large-vessel disease.

There was no significant difference in acute phase SSS score between the LVD and the cryptogenic stroke group (table 1). Furthermore, there were no significant correlations between the SSS score and the C3 or C3a levels, except for an inverse correlation between this score and the C3 level at follow-up in the LVD group (table 2). In contrast, in both the cryptogenic and the LVD group, we obtained an inverse correlation between the acute phase SSS score and the acute hsCRP levels $(\rho=-0.41, p<0.001$, and $\rho=-0.36, p=0.002$, respectively) but not between the acute phase SSS score and the follow-up hsCRP levels.
Association of Plasma C3 and C3a Levels with Stroke

Detailed results of binary logistic regression analysis of the case/control status are presented in table 3 and values of cutoffs for C3 and C3a tertiles are listed in table 4 . Acute phase plasma $\mathrm{C} 3$ concentration in the upper and middle third was associated with stroke in cryptogenic group both in the univariate and multivariate analysis (adjusted for age, sex, smoking status, hypertension and diabetes). In the LVD group, acute phase plasma C3 levels in the upper third were associated with stroke in both models. Follow-up plasma C3 levels in the upper third were associated with stroke in the cryptogenic group only 
Table 4. Cutoffs for tertiles of plasma C3 and C3a levels

\begin{tabular}{|c|c|c|c|c|}
\hline & \multicolumn{2}{|c|}{ Cryptogenic stroke } & \multicolumn{2}{|l|}{ LVD stroke } \\
\hline & acute & follow-up & acute & follow-up \\
\hline \multicolumn{5}{|l|}{$\mathrm{C} 3, \mathrm{mg} / \mathrm{l}$} \\
\hline Lower third & $<467.88$ & $<451.70$ & $<320.46$ & $<316.78$ \\
\hline Middle third & $467.88-566.67$ & $451.70-557.21$ & $320.46-450.27$ & $316.78-391.52$ \\
\hline Upper third & $>566.67$ & $>557.21$ & $>450.27$ & $>391.518$ \\
\hline \multicolumn{5}{|l|}{$\mathrm{C} 3 \mathrm{a}, \mu \mathrm{g} / \mathrm{l}$} \\
\hline Lower third & $<100.58$ & $<87.69$ & $<171.78$ & $<152.07$ \\
\hline Middle third & $100.58-175.22$ & 87.69-131.11 & $171.78-260.28$ & $152.07-220.27$ \\
\hline Upper third & $>175.22$ & $>131.11$ & $>260.28$ & $>220.27$ \\
\hline
\end{tabular}

LVD = Large-vessel disease.

Table 5. Selected ${ }^{1}$ univariate and multivariate ORs with 95\% CIs of association between C3 and C3a levels and functional outcome

\begin{tabular}{|c|c|c|c|c|c|}
\hline \multicolumn{2}{|l|}{ Tertiles of C3 and C3a } & \multirow{2}{*}{$\begin{array}{l}\text { Favorable } \\
\text { outcome, } \mathrm{n}\end{array}$} & \multirow{2}{*}{$\begin{array}{l}\text { Unfavorable } \\
\text { outcome, } \mathrm{n}\end{array}$} & \multirow{2}{*}{$\begin{array}{l}\text { Univariate } \\
\text { OR }\end{array}$} & \multirow{2}{*}{$\begin{array}{l}\text { Multivariate model } 2 \\
\text { OR }\end{array}$} \\
\hline \multirow{5}{*}{$\begin{array}{l}\text { Outcome at } 3 \text { months } \\
\text { Cryptogenic stroke }\end{array}$} & & & & & \\
\hline & C3a acute, all & 47 & 28 & & \\
\hline & Lower third & 15 & 4 & 1.0 (reference) & 1.0 (reference) \\
\hline & Middle third & 17 & 9 & $1.99(0.51-7.79)$ & $1.97(0.50-7.76)$ \\
\hline & Upper third & 15 & 15 & $3.75(1.01-13.96)^{*}$ & $3.74(0.99-14.04)$ \\
\hline \multirow[t]{4}{*}{ LVD stroke } & C3 follow-up, all & 47 & 17 & & \\
\hline & Lower third & 17 & 2 & 1.0 (reference) & 1.0 (reference) \\
\hline & Middle third & 14 & 5 & $3.04(0.51-18.10)$ & $3.25(0.53-20.04)$ \\
\hline & Upper third & 16 & 10 & $5.31(1.01-28.07)^{*}$ & $5.56(1.03-29.93)^{*}$ \\
\hline \multicolumn{6}{|l|}{ Outcome at 2 years } \\
\hline \multirow[t]{4}{*}{ Cryptogenic stroke } & C3a acute, all & 52 & 22 & & \\
\hline & Lower third & 16 & 3 & 1.0 (reference) & 1.0 (reference) \\
\hline & Middle third & 18 & 8 & $2.37(0.53-10.50)$ & $2.35(0.51-10.71)$ \\
\hline & Upper third & 18 & 11 & $3.25(0.76-13.80)$ & $3.15(0.73-13.69)$ \\
\hline \multirow[t]{4}{*}{ LVD stroke } & C3 follow-up, all & 44 & 21 & & \\
\hline & Lower third & 16 & 3 & 1.0 (reference) & 1.0 (reference) \\
\hline & Middle third & 14 & 5 & $1.90(0.38-9.44)$ & $1.75(0.35-8.82)$ \\
\hline & Upper third & 14 & 13 & $4.95(1.17-21.03)^{*}$ & $4.75(1.11-20.30)^{*}$ \\
\hline
\end{tabular}

Figures shown in parentheses are $95 \%$ CIs.

${ }^{*} \mathrm{p}<0.05$. OR = Odds ratio; CI = confidence interval; LVD = large-vessel disease. Model 2: adjusted for age and sex.

${ }^{1}$ Remaining nonsignificant results are not shown.

in the univariate model, whereas plasma C3 levels at this time point in the LVD group were associated with stroke in both regression models.

Higher acute C3a levels were also associated with stroke in both subtypes under the unadjusted as well as under the multiple-adjusted model. At 3 months after stroke, elevated C3a plasma concentration was associated with case/control status only in the LVD group. This association was independent of traditional risk factors.

\section{Association of Plasma C3 and C3a Levels with}

Functional Outcome

Among the investigated cryptogenic stroke patients, $28(37 \%)$ and $22(30 \%)$ had an unfavorable outcome (mRS 
$3-6)$ at 3 months and at 2 years after the stroke event, respectively. In the LVD stroke group, 17 patients (27\%) and 21 patients $(32 \%)$ had an unfavorable outcome at the 3-month and 2-year follow-up, respectively. The acutephase plasma C3a levels in the upper third were found to be associated with an increased risk of unfavorable outcome at 3 months in the cryptogenic stroke group in the univariate analysis. However, this association was lost after adjustment for age and sex. In the LVD group, plasma C3 levels in the upper third at the 3-month follow-up were associated with an unfavorable outcome after 3 months as well as after 2 years both in the univariate analysis and after adjustment for age and sex (table 5).

\section{Discussion}

Published reports on systemic complement activation after ischemic stroke have been based on studies involving small patients groups that did not allow for a separate analysis of complement system response in individual etiologic subtypes of ischemic stroke [18, 22]. Furthermore, there is only a limited number of reports on associations between complement levels and clinical outcome. In the present study, we have found that both in cryptogenic and LVD stroke, plasma levels of both C3 and C3a were elevated in the acute phase and were associated with ischemic stroke also after adjustment for the influence of traditional risk factors (age, sex, smoking, hypertension and diabetes). These results confirm previous findings showing elevated plasma C3a levels during the first 7 days after ischemic stroke [18] and suggest that systemic complement activation in the early poststroke phase is an important contributor to the inflammatory response after ischemic stroke. This notion is supported by a previous study that found increased plasma levels of C5b-9 during the first 12 days after ischemic stroke [22]. In both stroke subtypes, $\mathrm{C} 3$ levels remained elevated also 3 months after stroke. In the LVD group, high plasma C3 levels at this time point showed an association with patient status independently of traditional risk factors. This finding points to the potential role of $\mathrm{C} 3$ in the pathophysiological mechanisms of ischemic stroke.

We observed differences between the stroke subtypes in terms of complement response. The plasma C3a and C3 levels showed a positive correlation only in the LVD group and only in the acute phase. Plasma C3a levels remained elevated at the follow-up only in the LVD group and showed a significant association with the casecontrol status. As atherosclerosis has been shown to be strongly associated with complement activation [23], it is conceivable that the elevation of plasma C3a levels at follow-up in the LVD group is at least partly a consequence of the activation of the complement cascade by the underlying pathophysiological processes. These data, together with our findings of a positive correlation between C3a and C3 in LVD but not in cryptogenic stroke, indicate that in our study, brain ischemia in the cryptogenic stroke patients was not caused by the acceleration of a less active and milder form of LVD, as proposed by Bang et al. $[24,25]$. Interestingly, plasma levels of hsCRP at the 3-month follow-up were associated with LVD but not other ischemic stroke subtypes [20], again pointing to substantial differences in the underlying mechanisms between LVD and cryptogenic stroke.

CRP and C3 are acute phase proteins produced in large amounts in the liver in response to injury. Our correlation data indicate that plasma levels of these two proteins may be altered differently in response to ischemic stroke. Plasma C3 concentration reflects the net effect of C3 synthesis (regulated by proinflammatory cytokines) and its consumption due to the activation of the complement cascade. Complement activation in this setting can be triggered by CRP and most likely other activators that are released following ischemic brain injury. Thus measurements of hsCRP, C3 and C3a after stroke provide complementary information on the patient's inflammatory status that might differ between strokes of different etiologies.

Elevated plasma levels of C3 exhibited an association with an unfavorable outcome at the 3-month follow-up as well as at 2 years after stroke. Follow-up but not acute levels of C3 were linked to an unfavorable functional outcome but only in the LVD group. This difference in the predictive value of plasma $\mathrm{C} 3$ levels in the two stroke subtypes may be due to the difference in the underlying conditions. In the LVD group, atherosclerosis leads to chronic complement activation and is a strong determinant of outcome. In the cryptogenic group, the cause of stroke is unknown and the extent of systemic inflammatory response to cerebral ischemia may play a lesser role in outcome determination. In the cryptogenic group, acute phase plasma C3a levels were associated with greater disability at the 3-month follow-up only in the univariate analysis and this association was lost after adjustment for age and sex. These findings are in line with data reported by Szeplaki et al. [16]. If infarct volume per se has an infuence on C3 levels, this may contribute to our findings of an association with outcome. However, somewhat unexpectedly and in contrast to hsCRP, we did not find any 
correlation between SSS score and plasma C3 or C3a levels in the acute phase. Although SSS score is only a crude measure of stroke severity that depends not only on infarct volume but also on its location, this finding speaks against any major effect of infarct size on these parameters.

Results from a number of animal studies point to a dual role of inflammation and complement activation in brain ischemia. Genetic deficiency of C3 was associated with larger cerebral infarct after experimental permanent focal brain ischemia [7]. On the other hand, inhibition of complement activation or C3 deficiency reduced infarct volume and neurological impairments after transient cerebral ischemia in rodents $[10-13,26]$. Blocking of $\mathrm{C} 3 \mathrm{a}$ binding to its receptor reduced infarct volume assessed $24 \mathrm{~h}$ after transient but not permanent cerebral ischemia in rodents [27]. Together with our findings, these results suggest that the role of complement proteins and their activation products in ischemic stroke may be rather complex and may depend on factors such as degree of reperfusion, stroke etiology and evaluation time point.

The present study has some limitations. As the SAHLSIS project was not initially designed to study inflammatory responses, a detailed history of recent infections or chronic inflammatory events was not obtained. Moreover, the design of our study does not allow for a comparison of the absolute magnitude of complement response between LVD and cryptogenic stroke patients. As we did not have access to direct measurements of infarct volume in the stroke patients, we cannot exclude that infarct volume confounded our results. It is important to note that the cryptogenic group in our study is not repre- sentative for all patients with cryptogenic stroke as patients with unfavorable outcomes are overrepresented in the present sample compared to the whole cryptogenic group in SAHLSIS. Due to low numbers of participants, adjustment only for age and sex was possible in the analysis of outcome. Therefore, larger studies controlling for the effect of infections, and enabling adjustment for infarct volume and additional risk factors are necessary.

In conclusion, our data suggest that plasma $\mathrm{C} 3$ and C3a levels are elevated in cryptogenic and LVD stroke. As high plasma C3 levels at 3 months after stroke were associated with unfavorable outcomes in LVD but not cryptogenic stroke patients, the predictive value of complement proteins and their derivatives may depend on the ischemic stroke subtype.

\section{Acknowledgements}

The authors acknowledge the excellent technical assistance of Mrs. Ingrid Eriksson. This study was supported by grants by the Swedish Research Council (grant No 20116 to M.P. and 14605 to C.J.), ALF Göteborg (grant 11267 to M.P. and 148861 to C.J.), the Swedish Stroke Foundation, the Swedish Heart and Lung Foundation (20100256), the Göteborg Foundation for Neurological Research, and the Y. Land's, J. and B. Wennerström's Foundations for Neurological Research and as well as the R. and T. Söderberg's, T. Nilsson's, Emelle's, E. Jacobson's, W. and M. Lundgren's, R. and U. Amlöv's Foundations.

\section{Disclosure Statement}

The authors declare that there are no conflicts of interest.

\section{References}

1 Lindsberg PJ, Grau AJ: Inflammation and infections as risk factors for ischemic stroke. Stroke 2003:34:2518-2532.

-2 Danton GH, Dietrich WD: Inflammatory mechanisms after ischemia and stroke. J Neuropathol Exp Neurol 2003;62:127-136.

-3 Stoll G, Bendszus M: Inflammation and atherosclerosis: novel insights into plaque formation and destabilization. Stroke 2006;37: 1923-1932.

4 Wahlgren CM, Zheng W, Shaalan W, Tang J, Bassiouny HS: Human carotid plaque calcification and vulnerability. Relationship between degree of plaque calcification, fibrous cap inflammatory gene expression and symptomatology. Cerebrovasc Dis 2009;27: 193-200.
5 Arenillas JF, Alvarez-Sabin J, Molina CA, Chacon P, Fernandez-Cadenas I, Ribo M, Delgado P, Rubiera M, Penalba A, Rovira A, Montaner J: Progression of symptomatic intracranial large artery atherosclerosis is associated with a proinflammatory state and impaired fibrinolysis. Stroke 2008;39:14561463.

6 Worthmann H, Tryc AB, Goldbecker A, Ma YT, Tountopoulou A, Hahn A, Dengler R, Lichtinghagen $\mathrm{R}$, Weissenborn $\mathrm{K}$ : The temporal profile of inflammatory markers and mediators in blood after acute ischemic stroke differs depending on stroke outcome. Cerebrovasc Dis 2010;30:85-92.
7 Rahpeymai Y, Hietala MA, Wilhelmsson U, Fotheringham A, Davies I, Nilsson AK, Zwirner J, Wetsel RA, Gerard C, Pekny M, Pekna M: Complement: a novel factor in basal and ischemia-induced neurogenesis. EMBO J 2006;25:1364-1374.

8 van Beek J, Nicole O, Ali C, Ischenko A, MacKenzie ET, Buisson A, Fontaine M: Complement anaphylatoxin C3a is selectively protective against NMDA-induced neuronal cell death. Neuroreport 2001;12:289293.

-9 Mukherjee P, Pasinetti GM: Complement anaphylatoxin $\mathrm{C} 5$ a neuroprotects through mitogen-activated protein kinase-dependent inhibition of caspase 3. J Neurochem 2001;77:43-49. 
10 De Simoni MG, Storini C, Barba M, Catapano L, Arabia AM, Rossi E, Bergamaschini L: Neuroprotection by complement (C1) inhibitor in mouse transient brain ischemia. J Cereb Blood Flow Metab 2003;23:232-239.

-11 Mocco J, Mack WJ, Ducruet AF, Sosunov SA, Sughrue ME, Hassid BG, Nair MN, Laufer I, Komotar RJ, Claire M, Holland H, Pinsky DJ, Connolly ES, Jr: Complement component C3 mediates inflammatory injury following focal cerebral ischemia. Circ Res 2006;99:209217.

-12 Huang J, Kim LJ, Mealey R, Marsh HC, Jr, Zhang Y, Tenner AJ, Connolly ES, Jr, Pinsky DJ: Neuronal protection in stroke by an sLexglycosylated complement inhibitory protein. Science 1999;285:595-599.

-13 Costa C, Zhao L, Shen Y, Su X, Hao L, Colgan SP, Stahl GL, Zhou T, Wang Y: Role of complement component C5 in cerebral ischemia/ reperfusion injury. Brain Res 2006;1100: 142-151.

14 Pedersen ED, Loberg EM, Vege E, Daha MR, Maehlen J, Mollnes TE: In situ deposition of complement in human acute brain ischaemia. Scand J Immunol 2009;69:555-562.

15 Lindsberg PJ, Ohman J, Lehto T, Karjalainen-Lindsberg ML, Paetau A, Wuorimaa T, Carpen O, Kaste M, Meri S: Complement activation in the central nervous system following blood-brain barrier damage in man. Ann Neurol 1996;40:587-596.
16 Szeplaki G, Szegedi R, Hirschberg K, Gombos T, Varga L, Karadi I, Entz L, Szeplaki Z, Garred P, Prohaszka Z, Fust G: Strong complement activation after acute ischemic stroke is associated with unfavorable outcomes. Atherosclerosis 2009;204:315-320.

17 Tamam Y, Iltumur K, Apak I: Assessment of acute phase proteins in acute ischemic stroke. Tohoku J Exp Med 2005;206:91-98.

18 Mocco J, Wilson DA, Komotar RJ, Sughrue ME, Coates K, Sacco RL, Elkind MS, Connolly ES, Jr: Alterations in plasma complement levels after human ischemic stroke. Neurosurgery 2006;59:28-33; discussion 28-33.

19 Jood K, Ladenvall C, Rosengren A, Blomstrand C, Jern C: Family history in ischemic stroke before 70 years of age: The Sahlgrenska Academy Study on Ischemic Stroke. Stroke 2005;36:1383-1387.

20 Ladenvall C, Jood K, Blomstrand C, Nilsson S, Jern C, Ladenvall P: Serum C-reactive protein concentration and genotype in relation to ischemic stroke subtype. Stroke 2006;37: 2018-2023.

21 von Elm E, Altman DG, Egger M, Pocock SJ, Gotzsche PC, Vandenbroucke JP: The strengthening the reporting of observational studies in epidemiology (STROBE) statement: guidelines for reporting observational studies. J Clin Epidemiol 2008;61:344349.
22 Pedersen ED, Waje-Andreassen U, Vedeler CA, Aamodt G, Mollnes TE: Systemic complement activation following human acute ischaemic stroke. Clin Exp Immunol 2004; 137:117-122.

-23 Szeplaki G, Varga L, Fust G, Prohaszka Z: Role of complement in the pathomechanism of atherosclerotic vascular diseases. Mol Immunol 2009;46:2784-2793.

24 Bang OY, Lee PH, Joo SY, Lee JS, Joo IS, Huh $\mathrm{K}$ : Frequency and mechanisms of stroke recurrence after cryptogenic stroke. Ann Neurol 2003;54:227-234.

25 Bang OY, Lee PH, Yeo SH, Kim JW, Joo IS, Huh K: Non-cardioembolic mechanisms in cryptogenic stroke: clinical and diffusionweighted imaging features. J Clin Neurol 2005; 1:50-58.

$>26$ Atkinson C, Zhu H, Qiao F, Varela JC, Yu J, Song H, Kindy MS, Tomlinson S: Complement-dependent P-selectin expression and injury following ischemic stroke. J Immunol 2006;177:7266-7274.

27 Ducruet AF, Hassid BG, Mack WJ, Sosunov SA, Otten ML, Fusco DJ, Hickman ZL, Kim GH, Komotar RJ, Mocco J, Connolly ES: C3a receptor modulation of granulocyte infiltration after murine focal cerebral ischemia is reperfusion dependent. J Cereb Blood Flow Metab 2008;28:1048-1058. 